\title{
BMJ Open Potentially disruptive life events: what are the immediate impacts on chronic disease management? A case-crossover analysis
}

\author{
Julie C Lauffenburger, ${ }^{1,2}$ Joshua J Gagne, ${ }^{1}$ Zirui Song, ${ }^{3}$ Gregory Brill, ${ }^{1}$ \\ Niteesh K Choudhry ${ }^{1,2}$
}

To cite: Lauffenburger JC, Gagne JJ, Song Z, et al. Potentially disruptive life events: what are the immediate impacts on chronic disease management?

A case-crossover analysis. BMJ Open 2016:6:e010958. doi:10.1136/bmjopen-2015010958

- Prepublication history and additional material is available. To view please visit the journal (http://dx.doi.org/ 10.1136/bmjopen-2015010958).

Received 23 December 2015 Revised 21 April 2016 Accepted 13 July 2016

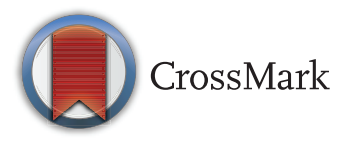

For numbered affiliations see end of article.

Correspondence to Dr Julie C Lauffenburger; jlauffenburger@bwh.harvard. edu

\section{ABSTRACT}

Objective: To explore the association between unexpected potentially disruptive life events in a patient or family member that may challenge an individual's ability to take medications as prescribed and the discontinuation of evidence-based medications for common, chronic conditions. Understanding the relationship between medication adherence and life stressors, especially those that can be identified using administrative data, may help identify patients at risk of non-adherence.

Design: Observational self-controlled case-crossover design.

Setting: Individuals in a nationally representative US commercial health insurance database.

Participants: Adult individuals who initiated an oral hypoglycaemic, antihypertensive and/or statin and subsequently stopped the medication for $\geq 90$ days.

Main outcome measure: Potentially disruptive life events among patients and their family members measured in the 30 days just before the medication was discontinued ('hazard period') compared with the 30 days before this period ('control period'). These events included personal injury, hospitalisation, emergency room visits, changes in insurance coverage, acute stress or acute anxiety.

Results: Among the 326519 patients meeting study criteria who discontinued their chronic disease medications, $88896(27.2 \%)$ experienced at least one potentially disruptive life event. Newly experiencing an injury (OR: $1.26,95 \% \mathrm{Cl} 1.12$ to 1.42 ), an emergency room visit (OR: 1.19, $95 \% \mathrm{Cl} 1.13$ to 1.26 ) and acute stress (OR: $1.19,95 \% \mathrm{Cl} 1.08$ to 1.31 ) were associated with discontinuation. Life events among patients' family members did not appear to be associated with medication discontinuation or occurred less frequently just prior to discontinuation.

Conclusions: Potentially disruptive life events among individuals identified using routinely collected claims data are associated with discontinuation of chronic disease medications. Awareness of these events may help providers or payers identify patients at risk of non-adherence to maximise patient outcomes.

\section{Strengths and limitations of this study}

- To the best of our knowledge, no study has used administrative claims to explore and characterise the association between potentially disruptive events in patients' lives or among their family members and discontinuing chronic medications.

- This study used a self-controlled case-crossover design in which each patient served as his or her own control, thereby controlling for measured and unmeasured factors that do not vary over time. This type of design can better approximate the causal process than other approaches.

- This is a study based on administrative data, and some bias is possible because of inadequately or incompletely measured event exposures. The case-crossover design may also be confounded by unmeasured time-varying patient characteristics, such as disease progression or socioeconomic changes.

- The results indicate that several life events appear to have immediate consequences that manifest in discontinuing chronic medications, particularly new personal injury, an acute stress reaction and visiting the emergency room. However, these results were intended to be exploratory and further research is warranted to validate these conclusions.

\section{INTRODUCTION}

Long-term adherence to evidence-based medications is poor. ${ }^{1}$ Fewer than half of patients continue to take medications for common chronic conditions, even within 1 year after initiating treatment. $^{2}{ }^{3}$ Since poor adherence is associated with potentially avoidable morbidity, mortality and healthcare costs, early identification and intervention in patients who are at risk of non-adherence is essential. $^{4-6}$ Even though a number of patient demographic, clinical and economic 
characteristics have been thought to affect adherence, many patients still stop taking their medications without a reason that is easily identifiable to physicians and other healthcare professionals. ${ }^{2}{ }^{7-9}$

Unexpected events, such as injuries, medical diagnoses or hospitalisations in a patient's life or a family member, may disrupt daily routines or an individual's engagement in his or her own care. ${ }^{10}$ For example, several small studies have suggested a negative impact of potentially stressful life events on medication adherence. $^{10-14}$ Identifying these events is difficult without direct and potentially time-consuming survey responses from patients. To the best of our knowledge, no study has used routinely collected administrative claims data to explore and characterise the influence of potentially disruptive events in patients' lives or those of family members on the risk that the patients discontinue evidence-based medications.

\section{METHODS}

\section{Study design and data source}

The Institutional Review Board of the Brigham and Women's Hospital approved this study; written informed consent was not obtained nor deemed to be required because the secondary administrative claims data were de-identified and anonymised prior to analysis. We used a self-controlled case-crossover design to examine the association between potentially disruptive life events and medication discontinuation. In this design, each patient serves as his or her own control at a different period in time, thereby controlling for measured and unmeasured confounding factors that do not vary over time. ${ }^{15} 16$

We used 2007-2012 data from UnitedHealthcare, which comprise a large nationwide administrative claims database of commercially insured individuals in the USA. The data were de-identified and anonymised patient-level claims for medical inpatient and outpatient procedures, hospitalisations, emergency room (ER) visits, office visits and outpatient pharmacy prescription drug dispensings linked to plan enrolment data. A family identifier, which was available on $\sim 10 \%$ of patients, was used to link patients with their family members. Family identifiers were available for some employer health plans; patients with and without identifiers did not appear to differ on any key demographic characteristics.

\section{Patient identification}

Our study cohort consisted of patients who initiated an oral antidiabetic, antihypertensive or cholesterollowering statin medication between 1 January 2008 and 30 June 2012. To be included, patients had to be $\geq 26$ years of age and have one or more drug claim and one or more healthcare claim and continuous enrolment in the 180-day baseline period before initiating a study medication. We constructed three disease-specific cohorts and excluded individuals if they had filled a medication for the disease of interest in the 180 days before initiation of a study drug. We selected an age cut-off of $\geq 26$ years to ensure that patients included in our cohort were not dependents on their parents' insurance plans.

The outcome of interest was discontinuation of the chronic disease medication after at least 60 days of continuous use. As only patients who experience the outcome contribute to the analysis of a case-crossover design, we restricted our cohort to individuals who discontinued their medication. We created a drug supply diary for each patient beginning on the date of their first prescription fill for a study drug (defined as their initiation date). We linked all prescription fills of the initiated medication based on dispensing date and days supply, where the supply for overlapping fills could accumulate to up to 180 days of excess supply. Switching was allowed among medications of the same chemically related therapeutic class (eg, sulfonylureas). Medication discontinuation was defined as failing to fill a new prescription within 90 days of finishing a previous prescription. The date of discontinuation was defined as the first day after the days supply elapsed, the first day of the 90-day discontinuation. In order to identify patients who had relatively consistent filling behaviour before discontinuation, we included only patients who ultimately discontinued treatment, filled two or more prescriptions, had $\geq 60$ days of medication supplied (including the first prescription) and had gaps without medication of 14 days or fewer between consecutive prescriptions.

\section{Measurement of potentially disruptive life events}

To assess events associated with discontinuation, potentially disruptive life events were measured in the 30 days before the drug discontinuation ('hazard period') and the 30 days immediately preceding the hazard period ('control period') in the medical and pharmacy claims (figure 1). These events were identified from factors in the Holmes and Rahe stress scale and were selected for their ability to be potentially measured in claims data and because they were acute exposures. ${ }^{17}$ We focused on patients who experienced the events of interest for the first time since initiating their medication, defined as those whose first incidence of each life event was within 60 days prior to discontinuing the medication (ie, within the hazard or control period); that is, we excluded patients who experienced the event between the initiation date and the first day of the control period.

The life events that were measured directly in individuals included personal injury and an ER visit. Indicators of potentially disruptive life events were also assessed, including an acute stress reaction or acute anxiety/panic attack. These events were defined according to the International Classification of Diseases 9th edition (ICD-9) codes, National Drug Codes (NDCs) or Current Procedural Therapy (CPT) codes as specified in online supplementary table S1. 


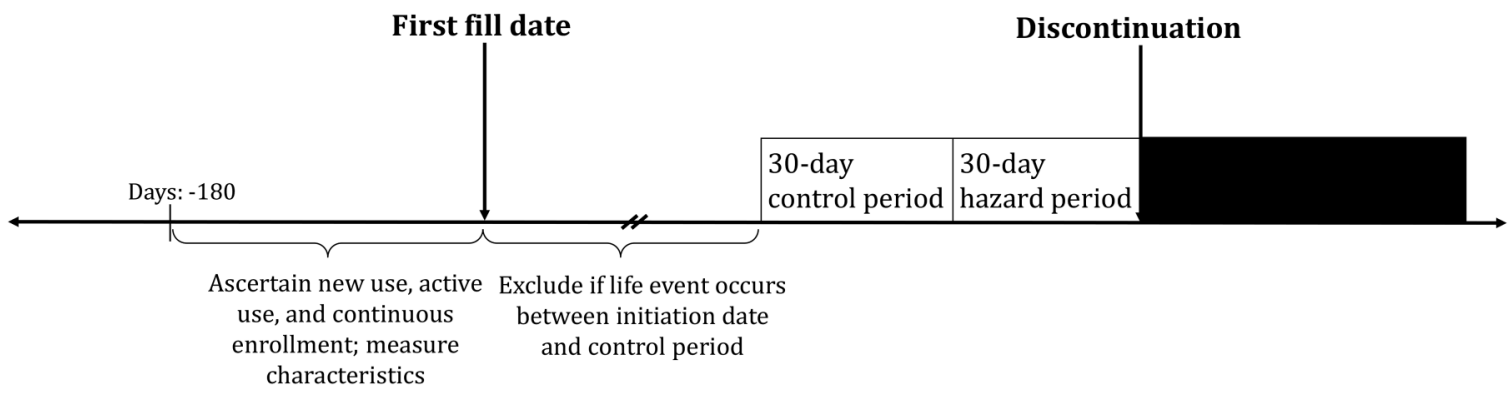

Figure 1 Schematic of the case-crossover design.

Potentially disruptive life events in the patients' immediate family members (eg, partner, child) were also measured among those with a family identifier that linked them with another insured beneficiary. These events included: changes in family member plan enrolment (ie, the addition or removal of a dependent other than at open enrolment), ER visit, hospitalisation, personal injury, acute stress and acute anxiety/panic attack (see online supplementary table S1).

\section{Baseline characteristics}

Patient demographic characteristics, including age and gender, were obtained from the enrolment files. Other characteristics were measured in the 6 months prior to medication initiation using ICD-9 or CPT codes, including comorbidities, combined comorbidity score (a measure of overall health status), ${ }^{18}$ the number of outpatient physician office visits and markers of healthy behaviours (colonoscopy and mammogram/prostate-specific antigen test).

\section{Statistical analysis}

The case-crossover design was implemented by comparing the frequency of each life event in the hazard period with the frequency of the event in the immediately preceding control period. ORs and 95\% CIs for the life events were estimated using separate conditional logistic regression models for each life event exposure. ${ }^{19-22}$ Patients only contributed to the analysis once if they initiated medications for multiple disease states and also met all of the other inclusion/exclusion criteria; in this situation, the medication discontinuation with the earliest initiation date was chosen for the analysis. By definition, patients with discordant periods of event occurrences contribute to the analysis (eg, an event occurring in the hazard period or the control period, but not both).${ }^{15}$ Conditional logistic regression analyses were also conducted among the subgroups of patients with at least one linked family member for the family member-specific life events. All analyses were conducted using SAS V.9.4 (SAS, Cary, North Carolina, USA).

\section{Sensitivity analyses}

We conducted multiple sensitivity analyses to assess the robustness of the results. First, we directly examined the frequency of events in patients before and after their last prescription fill by restricting analyses to those whose last prescription fill was 30 days; in other words, in these patients, their last prescription was filled on the first day of the hazard period. Second, we restricted to patients who had filled $\geq 180$ days of medication to examine the impact of the life events among longer term users. Third, we stratified the analyses by time since initiation, separately examining individuals with filling histories less than and greater than the mean time until discontinuation for each life event. Fourth, we restricted to statin initiators, because switching across drug classes is uncommon. Fifth, we measured the life events only in the inpatient data, when applicable. Finally, we also modified the inclusion criteria for the cohort in two different ways. We extended the discontinuation definition to 180 days after the end of a patient's accumulated supply of medications. In addition, we increased the 'hazard' and 'control' periods to the 60 days before the drug discontinuation ('hazard period') and the 60 days immediately preceding the hazard period ('case period') among patients with at least 120 days of filling behaviour.

\section{RESULTS}

Our study cohort consisted of 326519 patients, of whom 54 072, 161225 and 137941 discontinued an oral hypoglycaemic, antihypertensive and/or statin, respectively (see online supplementary table S2). Among these patients, the mean age was 52.1 years, and $46.9 \%$ were men. Patients had a mean combined comorbidity score of 0.1 (SD: 1.2) (table 1). On average, patients discontinued taking their chronic medications after 261 days (SD: 269), with a median of 151 days.

\section{Individual life events}

Among the patients in our cohort, 88896 (27.2\%) experienced at least one potentially disruptive life event (table 2). Of these, $18194(20.5 \%)$ patients first experienced the life event in the 60 days prior to medication discontinuation. In total, $8767(48.2 \%)$ patients experienced an event in the control period and not the hazard period, and 9157 (50.3\%) patients incurred an event in the hazard period and not the control period. The case-crossover analyses ultimately consisted of these patients with discordant event periods. 
Table 1 Baseline characteristics of the study participants

\begin{tabular}{|c|c|c|c|c|}
\hline Characteristics, $\mathbf{N}(\%)$ & $\begin{array}{l}\text { All patients* } \\
(\mathrm{N}=326519)\end{array}$ & $\begin{array}{l}\text { Antidiabetic } \\
\text { discontinuers } \\
(\mathrm{N}=54072)\end{array}$ & $\begin{array}{l}\text { Antihypertensive } \\
\text { discontinuers } \\
\text { ( } \mathrm{N}=161225)\end{array}$ & $\begin{array}{l}\text { Statin } \\
\text { discontinuers } \\
(\mathrm{N}=137 \text { 941) }\end{array}$ \\
\hline Age, mean (SD) & $52.1(11.6)$ & $50.9(12.4)$ & $51.0(12.0)$ & $54.3(10.4)$ \\
\hline Male gender & $153118(46.9)$ & $23773(44.0)$ & 72329 (44.9) & $71317(51.7)$ \\
\hline Coronary artery disease & $29167(8.9)$ & $4672(8.6)$ & $12770(7.9)$ & $15356(11.1)$ \\
\hline Hypertension & $146394(44.8)$ & 26860 (49.7) & $69786(43.3)$ & 65009 (47.1) \\
\hline Congestive heart failure & $6674(2.1)$ & $1382(2.6)$ & $3195(2.0)$ & $2993(2.2)$ \\
\hline Stroke or transient ischaemic attack & $6882(2.1)$ & $939(1.7)$ & 3131 (1.9) & $3634(2.6)$ \\
\hline Diabetes & $96754(29.6)$ & $28272(52.3)$ & $25838(16.0)$ & $30295(22.0)$ \\
\hline Depression & $26005(8.0)$ & $3957(7.3)$ & $13355(8.3)$ & $10747(7.8)$ \\
\hline Chronic obstructive pulmonary disease/asthma & $27491(8.4)$ & $4779(8.8)$ & $13847(8.6)$ & $11373(8.2)$ \\
\hline Liver disease & $7696(2.4)$ & $1622(3.0)$ & 3772 (2.3) & $2931(2.1)$ \\
\hline Chronic kidney disease & $7962(2.4)$ & $1883(3.5)$ & $3475(2.2)$ & $3486(2.5)$ \\
\hline Alzheimer's/dementia & $1909(0.6)$ & $286(0.5)$ & $1026(0.6)$ & $804(0.6)$ \\
\hline Comorbidity score, mean (SD) & $0.1(1.2)$ & $0.1(1.2)$ & $0.1(1.2)$ & $0.1(1.1)$ \\
\hline No. office visits, mean (SD) & $6.6(7.7)$ & $7.1(8.0)$ & $6.8(8.1)$ & $6.2(7.1)$ \\
\hline Colonoscopy & $13184(4.0)$ & $1831(3.4)$ & $6348(3.9)$ & $6093(4.4)$ \\
\hline Mammogram/PSA test & $56975(17.5)$ & $8662(16.0)$ & $23334(14.5)$ & $29670(21.5)$ \\
\hline Time until discontinuation, mean (SD) & 261 (269) & $270(271)$ & $266(271)$ & $271(280)$ \\
\hline
\end{tabular}

Table 2 Association between newly experiencing potentially disruptive life events and medication discontinuation

\begin{tabular}{|c|c|c|c|c|c|}
\hline Event & $\begin{array}{l}\text { Control } \\
\text { (N) }\end{array}$ & $\begin{array}{l}\text { Hazard } \\
\text { (N) }\end{array}$ & $\begin{array}{l}\text { Discordant } \\
\text { pairs }(\mathrm{N})\end{array}$ & $\begin{array}{l}\text { Time until } \\
\text { discontinuation, } \\
\text { mean (SD)* }\end{array}$ & OR $(95 \% \mathrm{Cl})$ \\
\hline \multicolumn{6}{|l|}{ Individual events } \\
\hline Personal injury & 737 & 871 & 1160 & $202(220)$ & $1.26(1.12$ to 1.42$)$ \\
\hline ER visit & 3434 & 3862 & 4938 & $181(200)$ & $1.19(1.13$ to 1.26$)$ \\
\hline Acute stress reaction & 1261 & 1411 & 1710 & $196(218)$ & $1.19(1.08$ to 1.31$)$ \\
\hline Acute anxiety/panic attack & 7030 & 6250 & 8588 & $151(172)$ & $0.83(0.80$ to 0.87$)$ \\
\hline \multicolumn{6}{|l|}{ Family events } \\
\hline Personal injury & 36 & 43 & 69 & $214(237)$ & $1.27(0.76$ to 2.12$)$ \\
\hline ER visit & 184 & 180 & 310 & $164(173)$ & $0.97(0.76$ to 1.24$)$ \\
\hline Hospitalisation & 596 & 503 & 1079 & $128(136)$ & $0.84(0.74$ to 0.95$)$ \\
\hline Acute stress reaction & 107 & 104 & 162 & $203(233)$ & 0.95 (0.66 to 1.37$)$ \\
\hline Acute anxiety/panic attack & 248 & 257 & 416 & $148(148)$ & $1.06(0.85$ to 1.31$)$ \\
\hline Addition to insurance plan & 911 & 666 & 1567 & $112(107)$ & $0.73(0.66$ to 0.81$)$ \\
\hline Removal from insurance plan & 130 & 141 & 271 & $171(205)$ & $1.09(0.86$ to 1.38$)$ \\
\hline
\end{tabular}

In the conditional logistic regression analyses of disruptive life events experienced by the patient themselves (table 2), personal injury (OR: $1.26,95 \%$ CI 1.12 to 1.42), ER visit (OR: $1.19,95 \%$ CI 1.13 to 1.26 ) and acute stress reaction (OR: $1.19,95 \%$ CI 1.08 to 1.31 ) were all more likely to occur in the hazard period compared with the control period. In contrast, patients were less likely to experience an acute anxiety/panic attack in the hazard than the control period (OR: $0.83,95 \%$ CI 0.80 to 0.87 ).

\section{Family member life events}

When examining the potential influence of family life events, $20832(6.4 \%)$ patients had an identifiable family member in the database (table 2). A family member hospitalisation (OR: $0.84,95 \%$ CI 0.75 to 0.95 ) and adding a new family member to their insurance plan (OR: 0.73 , $95 \%$ CI 0.66 to 0.81 ) were less likely to occur in the hazard period versus the control period. No other events were significantly associated with an increased odds of discontinuation.

\section{Sensitivity analyses}

The results of our sensitivity analyses are presented in table 3 and online supplementary table S3 for individual and family life events. Overall, increasing the time that had to elapse before defining a patient as having 
discontinued treatment (from 90 to 180 days), restricting to statin initiators, measuring only inpatient events and restricting to only patients whose last prescription was filled on the first day of the hazard period did not materially change the results.

In contrast, increasing the minimum number of days of medication that patients must have filled before discontinuation from 60 to 180 days generally increased the ORs for the association between discontinuation and individual events. In particular, patients were more likely to experience acute anxiety in the hazard period prior to discontinuing with this longer medication use requirement (OR: 1.14, 95\% CI 1.03 to 1.25 ). When stratifying on the mean time until discontinuing (151 days for acute anxiety), we found a similar association (OR: 1.09, 95\% CI 1.01 to 1.19) among those who had used the medication for at least 151 days. A stronger association between acute stress and discontinuation was also noted when restricting to those with medication use longer than the mean time (196 days) (OR: 1.58, 95\% CI 1.31 to 1.91 ).

\section{DISCUSSION}

To the best of our knowledge, this is the first study to examine the link between potentially disruptive events in the lives of a large number of individuals and medication adherence using routinely collected administrative claims. We found that several life events appear to be associated with discontinuing chronic essential medications. In particular, a new diagnosis of personal injury or an acute stress reaction and visiting the ER are associated with patients discontinuing therapy. We also found that the longer the patients were on the medication, the higher the frequency of the life events just prior to discontinuation, implying that the relative influence of disruptive life events may be stronger in patients who demonstrate better adherence to their medications perhaps because they had fewer other barriers to adherence. We found no evidence that life events in family members' lives occurred more frequently just prior to medication discontinuation than during a similar interval in patients' previous medication use history.

Many patient demographic, clinical and economic characteristics have been shown to be associated with poor adherence and lead to medication discontinuation. ${ }^{2}{ }^{7-9}$ Despite this, the ability to identify factors associated with medication non-adherence is frequently limited, suggesting that other events in patients' lives are affecting their ability to sustain their medication-taking behaviours. ${ }^{23-25}$ Previous research on the impact of these types of life stressors on medication adherence has primarily been through the use of patient surveys in a small number of studies. For instance, among 406 patients with hypertension and myocardial infarction, poor self-reported medication adherence was associated with an $86 \%$ increased odds of perceived life chaos. ${ }^{12}$ Similarly, a high self-reported life events score was

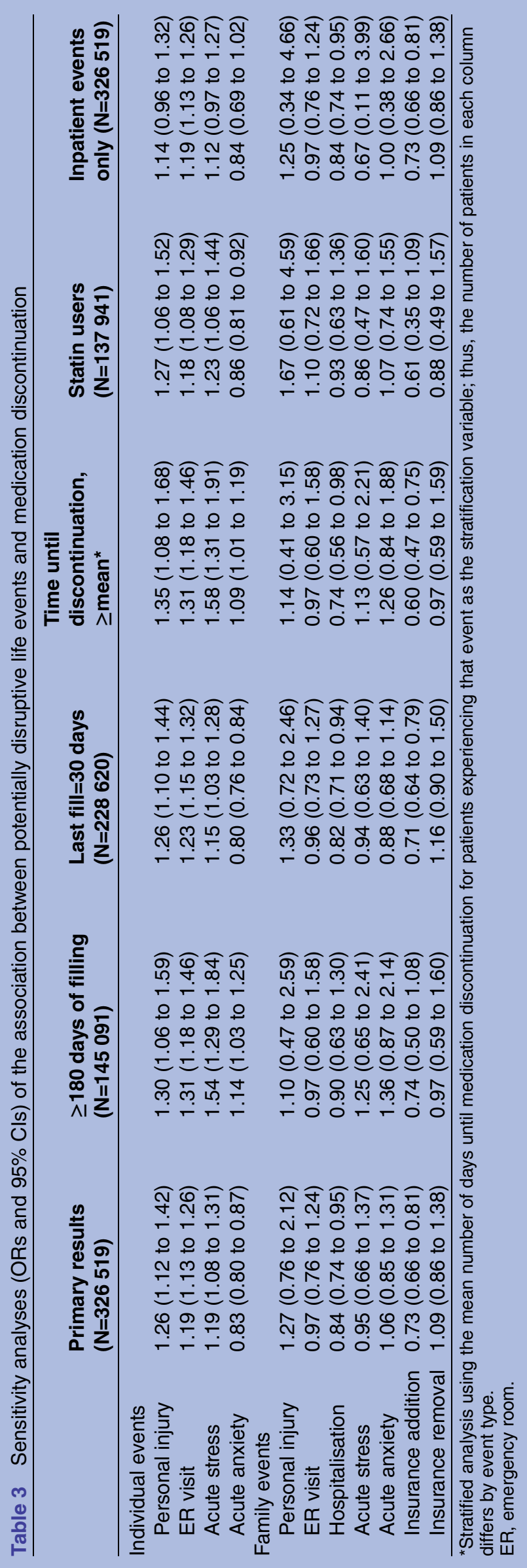


associated with a $68 \%$ decreased odds of being adherent to antihypertensive medications among 1965 older adults. ${ }^{14}$ Our results build on these previous findings by identifying additional specific events beyond other previously identified characteristics that could be associated with medication discontinuation. By relying on claims data rather than patient self-report, this study also highlights the disruptive influence of life events even among patients who may not be aware of, let alone be able to express, their underlying life chaos or non-adherence.

The association between disruptive life events and discontinuing essential medications highlights some noteworthy implications for patient care. Without intervention, these life stressors could result in increased morbidity and healthcare spending from preventable complications from poor medication use. These life events seem to reflect life chaos that is particularly influential upon patient's first exposure to a stressor. For example, a personal injury or other event leading to an ER visit appears to be sufficiently disruptive to be associated with subsequent medication discontinuation.

A few life events occurred less frequently just prior to medication discontinuation, including acute anxiety in the individual and addition to insurance and hospitalisation among family members. There are several potential explanations for these and other null findings. First, it is possible that some of these events may not be so distracting as to cause abrupt discontinuation and may instead lead to more minor, but still potentially impactful, unmeasured changes in adherence patterns. Second, some events among family members may take longer to manifest into larger problems for an individual, and there may be spillover effects of an event into later periods of time. Third, it is also possible that these events, such as a family member being hospitalised, could improve adherence. Finally, family member life events were measured indirectly, and life chaos could most strongly affect the individual rather than the nuclear family; however, the sample size for some of the events was low, with wide CIs.

These findings suggest several possible interventions relevant to patients and providers. In principle, one strategy to identify life chaos and stressors may be to include these additional life events among other tools that are used to predict patient non-adherence. Using these markers may further identify patients who are at risk of discontinuation. As administrative claims data are becoming increasingly shared between providers, the ability to prospectively identify patients who are facing life stressors will likely increase. Insurers may also find these routinely collected markers to be useful in identifying patients for disease management programmes. Future studies should consider including some of these potentially disruptive life events to help identify patients at risk of discontinuation and who may be in need of intervention. Further exploration into other markers of life chaos and its impacts on medication-taking is essential.
Our findings should be considered in light of several limitations. First, this is a study based on administrative data, and some bias is possible because of inadequately or incompletely measured event exposures. However, the misclassification would be unlikely to differ between the hazard and control periods. Given the nature of the data, medication discontinuation was also measured indirectly using a similar method from previous research. ${ }^{20} \quad 26$ Though our approach was more conservative, we may not have captured the full impact on medication-taking behaviour by only studying complete discontinuation. In the primary analysis, it is also possible that true discontinuation happened before the events; however, the sensitivity analysis in which we restricted to those whose last fill was 30 days addresses this potential concern, and the results are virtually the same. The case-crossover design may also be confounded by unmeasured time-varying patient characteristics, such as disease progression or socioeconomic changes. However, we chose a control period directly adjacent to the hazard period to mitigate this issue. It is also possible that some events have longer latency periods before being they are diagnosed or measured, but we purposely focused on immediate, discrete events and markers. These life events could also have led to clinically appropriate medication discontinuation, rather than patient-initiated medication non-adherence. Finally, despite being a large, nationwide sample, some estimates had wide $95 \%$ CIs due to a small number of events and family identifiers only being available for $10 \%$ of the cohort.

\section{CONCLUSION}

Disruptive life events among individuals that are identified using routinely collected claims data are associated with a higher risk of treatment discontinuation. Prospectively ascertaining these events may help to identify patients who are in need of near-term efforts to maintain medication adherence. Future efforts should continue to identify these markers of life chaos in patients at risk of discontinuing their medications.

\section{Author affiliations}

${ }^{1}$ Division of Pharmacoepidemiology and Pharmacoeconomics, Department of Medicine, Brigham and Women's Hospital and Harvard Medical School,

Boston, Massachusetts, USA

${ }^{2}$ Department of Medicine, Center for Healthcare Delivery Sciences, Brigham and Women's Hospital, Boston, Massachusetts, USA

${ }^{3}$ Department of Medicine, Massachusetts General Hospital, Boston, Massachusetts, USA

Contributors All authors had access to study results and contributed meaningfully to the analysis. JCL, JJG, ZS and NKC contributed to the study conception and design and interpretation of results. JCL and NKC contributed to manuscript drafting. GB prepared and analysed the data. JJG and ZS provided interpretation of results and critical manuscript revisions.

Competing interests None declared

Ethics approval Brigham and Women's Hospital.

Provenance and peer review Not commissioned; externally peer reviewed. 
Data sharing statement Owing to the Data Use Agreements with the health insurer, the raw data cannot be shared publicly. However, additional data are available in the supplemental file.

Open Access This is an Open Access article distributed in accordance with the Creative Commons Attribution Non Commercial (CC BY-NC 4.0) license, which permits others to distribute, remix, adapt, build upon this work noncommercially, and license their derivative works on different terms, provided the original work is properly cited and the use is non-commercial. See: http:// creativecommons.org/licenses/by-nc/4.0/

\section{REFERENCES}

1. Osterberg L, Blaschke T. Adherence to medication. N Engl J Med 2005;353:487-97.

2. Choudhry NK, Setoguchi S, Levin R, et al. Trends in adherence to secondary prevention medications in elderly post-myocardial infarction patients. Pharmacoepidemiol Drug Saf 2008;17:1189-96.

3. Lauffenburger JC, Robinson JG, Oramasionwu C, et al. Racial/ ethnic and gender gaps in the use of and adherence to evidencebased preventive therapies among elderly Medicare Part $D$ beneficiaries after acute myocardial infarction. Circulation 2014;18: 754-63.

4. Choudhry NK, Glynn RJ, Avorn J, et al. Untangling the relationship between medication adherence and post-myocardial infarction outcomes: medication adherence and clinical outcomes. Am Heart $J$ 2014;167:51-8.e5.

5. Bitton A, Choudhry NK, Matlin OS, et al. The impact of medication adherence on coronary artery disease costs and outcomes: a systematic review. Am J Med 2013;126:357.e7-e27.

6. Sokol MC, McGuigan KA, Verbrugge RR, et al. Impact of medication adherence on hospitalization risk and healthcare cost. Med Care 2005;43:521-30.

7. Ho PM, Spertus JA, Masoudi FA, et al. Impact of medication therapy discontinuation on mortality after myocardial infarction. Arch Intern Med 2006;166:1842-7.

8. Baroletti S, Dell'Orfano H. Medication adherence in cardiovascular disease. Circulation 2010;121:1455-8.

9. Gellad WF, Grenard JL, Marcum ZA. A systematic review of barriers to medication adherence in the elderly: looking beyond cost and regimen complexity. Am J Geriatr Pharmacother 2011;9:11-23.

10. Mugavero MJ, Raper JL, Reif S, et al. Overload: impact of incident stressful events on antiretroviral medication adherence and virologic failure in a longitudinal, multisite human immunodeficiency virus cohort study. Psych Med 2009;71:920-6.

11. Wong MD, Sarkisian CA, Davis C, et al. The association between life chaos, health care use, and health status among HIV-infected persons. J Gen Intern Med 2007;22:1286-91.
12. Zullig LL, Shaw RJ, Crowley MJ, et al. Association between perceived life chaos and medication adherence in a postmyocardial infarction population. Circ Cardiovasc Qual Outcomes 2013;6: 619-25.

13. Crowley MJ, Zullig LL, Shah BR, et al. Medication non-adherence after myocardial infarction: an exploration of modifying factors. $J$ Gen Intern Med 2015;30:83-90.

14. Krousel-Wood M, Joyce C, Holt E, et al. Predictors of decline in medication adherence: results from the cohort study of medication adherence among older adults. Hypertension 2011;58:804-10.

15. Maclure $\mathrm{M}$. The case-crossover design: a method for studying transient effects on the risk of acute events. Am J Epidemiol 1991:133:144-53

16. Maclure M, Mittleman MA. Should we use a case-crossover design? Annu Rev Public Health 2000;21:193-221.

17. Holmes TH, Rahe RH. The social readjustment rating scale. $J$ Psychosom Res 1967;11:213-18.

18. Gagne JJ, Glynn RJ, Avorn J, et al. A combined comorbidity score predicted mortality in elderly patients better than existing scores. J Clin Epidemiol 2011;64:749-59.

19. Mittleman MA, Maclure M, Robins JM. Control sampling strategies for case-crossover studies: an assessment of relative efficiency. Am J Epidemiol 1995;142:91-8.

20. Brookhart MA, Patrick AR, Schneeweiss S, et al. Physician follow-up and provider continuity are associated with long-term medication adherence: a study of the dynamics of statin use. Arch Intern Med 2007;167:847-52.

21. Gagne JJ, Avorn J, Shrank WH, et al. Refilling and switching of antiepileptic drugs and seizure-related events. Clin Pharmacol Ther 2010;88:347-53.

22. Kesselheim AS, Bykov $\mathrm{K}$, Avorn J, et al. Burden of changes in pill appearance for patients receiving generic cardiovascular medications after myocardial infarction: cohort and nested case-control studies. Ann Intern Med 2014:161:96-103.

23. Chan DC, Shrank WH, Cutler D, et al. Patient, physician, and payment predictors of statin adherence. Med Care 2010;48:196-202

24. Raebel MA, Ellis JL, Carroll NM, et al. Characteristics of patients with primary non-adherence to medications for hypertension, diabetes, and lipid disorders. J Gen Intern Med 2012;27:57-64.

25. Franklin JM, Shrank WH, Lii J, et al. Observing versus predicting: initial patterns of filling predict long-term adherence more accurately than high-dimensional modeling techniques. Health Serv Res 2016;51:220-39.

26. Sattler EL, Lee JS, Perri M III. Medication (re)fill adherence measures derived from pharmacy claims data in older Americans: a review of the literature. Drugs Aging 2013;30: 383-99. 